\title{
Abundances of Organic Molecules in Molecular Cloud Cores
}

\author{
Edmund C. Sutton \\ University of Illinois, 1002 W. Green St., Urbana, IL 61801, USA
}

Andrej M. Sobolev

Astronomical Observatory, Ural State University, Lenin Street 51, Ekaterinburg 620083, Russia

\begin{abstract}
Molecular cloud cores are often found to contain regions with high abundances of organic molecules such as formaldehyde, methanol, ethanol, dimethyl ether, and methyl formate. First we will review the status of observations of these molecules in a number of sources and discuss some of the limitations of present techniques. Then we will discuss systematic factors involved in the conversion of column densities into fractional abundances and introduce an independent method of calibrating that conversion. Finally we will present recent results from high spatial resolution observations of $\mathrm{W} 3(\mathrm{OH})$.
\end{abstract}

\section{Molecular Column Densities}

Organic molecules such as formaldehyde, methanol, ethanol, dimethyl ether, and methyl formate are now routinely observable in a variety of star formation regions. Selected results of observations with single dish radio telescopes are summarized in Table 1, which shows that fractional abundances of these molecules (relative to molecular hydrogen) are generally of order $10^{-9}$ to $10^{-6}$.

Calculation of a fractional abundance begins with determining the molecular column density along the line of sight. An observation of a single spectral line provides limited information, relating to the populations in the upper and lower energy levels of the observed line. Extrapolation to the total molecular column density requires assumptions, which may introduce uncertainties. Often such assumptions lead to underestimates of the molecular column densities.

For example, consider an observation of a line at some moderate energy above the ground state. One can assume a Boltzmann distribution at a particular excitation temperature in order to estimate the populations of the other energy levels. However, it is always possible that there is, in addition, some amount of very cold gas in which the observed energy levels are not well populated. This gas would effectively be missed by the observations. Similarly a region of very warm gas would populate primarily the very high energy levels and would be similarly missed by the observations. The obvious remedy is to observe low, medium, and high excitation lines in order to be able to see departures from the simple model of molecular excitation. 
A similar situation arises with regards to opacity. Observations of a single line can be interpreted to give a column density under the assumption that the line is optically thin. But if the line is optically thick, one will effectively miss a large fraction of the molecules, those hidden behind the region where the line becomes optically thick. Here the remedy is to observe lines with a wide range of intrinsic line strengths. If at least some of the lines are optically thin, one can model the behavior of the optically thick lines and reach a reliable estimate of the total column density.

Table 1. Log Fractional Abundances

\begin{tabular}{lrrrrrr}
\hline Source & $\mathrm{H}_{2} \mathrm{CO}$ & $\mathrm{CH}_{3} \mathrm{OH}$ & $\mathrm{C}_{2} \mathrm{H}_{5} \mathrm{OH}$ & $\mathrm{CH}_{3} \mathrm{OCH}_{3}$ & $\mathrm{HCOOCH}_{3}$ & Ref. \\
\hline Orion-KL $^{a}$ & -7.8 & -6.9 & & -8.0 & -8.1 & 1 \\
& -7.4 & -6.4 & -8.8 & -7.7 & -7.5 & 4 \\
& & -6.7 & & & -8.2 & 12 \\
Sgr B2(N) & & & & -8.3 & & 2 \\
& $>-9.3$ & -6.7 & -9.0 & -8.5 & -9.0 & 10 \\
$\mathrm{~W} 3\left(\mathrm{OH} / \mathrm{H}_{2} \mathrm{O}\right)$ & -8.4 & -7.0 & & -7.7 & -8.2 & 8 \\
& -9.0 & -7.7 & & & & 3 \\
& & -7.4 & & & & 12 \\
G34.3+0.15 & $>-9.5$ & -7.2 & -8.2 & & -7.5 & 7 \\
& & -7.1 & -8.4 & -8.0 & & 9 \\
& & -7.0 & -8.2 & -8.0 & -8.3 & 12 \\
IRAS 16293-2422 & -9.2 & -8.4 & -9.7 & & & 5 \\
NGC 1333 & $-8.7^{b}$ & & & & & \\
& $-6.4^{c}$ & $-5.0^{c}$ & & & & 6 \\
G327.3-0.6 & $>-8.2$ & -7.3 & -8.6 & -6.5 & -7.3 & 11 \\
& & -7.0 & -8.1 & -8.0 & & 9 \\
& & -7.0 & -8.0 & -7.5 & & 12 \\
NGC 6334 I & & -6.7 & -8.2 & -7.7 & & 9 \\
& & -6.7 & -8.0 & -7.4 & & 12 \\
\hline \hline
\end{tabular}

References.- (1) Blake et al. 1987. (2) Sutton et al. 1991. (3) Helmich et al. 1994. (4) Sutton et al. 1995. (5) van Dishoeck et al. 1995. (6) Blake et al. 1995. (7) Macdonald et al. 1996. (8) Helmich \& van Dishoeck 1997. (9) Nummelin et al. 1998. (10) Nummelin et al. 2000. (11) Gibb et al. 2000. (12) Ikeda et al. 2001.

${ }^{a}$ compact ridge source.

${ }^{b}$ IRAS4b core.

${ }^{c}$ IRAS4b wing. 
This is essentially what is done with the rotation diagram technique (e.g. Blake et al. 1987; Goldsmith \& Langer 1999). A large number of lines are observed, and the logarithms of their intensities (normalized by the line strengths) are plotted against their upper state energies. The slope of a straight line fitted to the data provides the excitation temperature, and the intercept is related to the column density. One might think that the rotation diagram technique breaks down when the assumptions of low optical depth or LTE excitation are violated. But in fact it provides the means for observing the effects of opacity and departures from LTE (Goldsmith \& Langer 1999; Sutton et al. 2003). The essential feature is sampling a large enough number of lines, spanning a sufficient range of energies and opacities.

\section{Fractional Abundances}

The standard method for converting molecular column densities into fractional abundances is to come up with an indirect estimate of the molecular hydrogen column density. One technique is to observe the dust continuum emission (assumed to be optically thin) and to relate it to molecular hydrogen using an assumed gas-to-dust ratio. An alternate technique is to observe an optically thin line of an isotopomer of $\mathrm{CO}$ and assume a fixed ratio of $\mathrm{CO}$ to $\mathrm{H}_{2}$. Both techniques seem to work and give reliable results, but both are based on indirect measurements of the molecular hydrogen column density.

Given the importance of this conversion factor, it is worth investigating whether there might be an independent method of checking the conversion. Hydrogen molecules make themselves known in molecular clouds primarily through collisions with other species. This is particularly true in the study of methanol maser excitation, where collisions tend to thermalize the population distribution of the methanol energy levels, quenching the maser mechanism. The results of Sutton et al. (2001) are that in the source W3 $(\mathrm{OH})$ the fractional abundance of methanol is in the range $10^{-6}$ to $10^{-5}$. This result is model dependent and not necessarily more precise than the standard technique for determining fractional abundances. But it is reassuring that it is in reasonable agreement with previous results and is based on an entirely independent determination of the abundance of molecular hydrogen.

Astrochemically this is important since methanol fractional abundances this high cannot be produced in the gas phase. Instead they are strong indications that methanol is present in dust grain mantles and is released into the gas phase by grain mantle evaporation (Caselli, Hasegawa, \& Herbst 1993).

\section{Improved Spatial Resolution}

Most fractional abundances measured to date are based on observations with single dish radio telescopes. However, results from millimeter interferometers are becoming available, and new instruments such as CARMA and ALMA will become increasingly important. Since there are likely to be variations in the spatial distributions of complex molecules, observations with high spatial resolution may reveal regions with higher and lower fractional abundances than presently observed. 
Here we report preliminary results of observations of methanol, methyl formate, and dimethyl ether in $\mathrm{W} 3(\mathrm{OH})$ at angular resolutions of about 2 arcsec $(0.02 \mathrm{pc})$ using the BIMA interferometer. For methanol we observed 38 lines and derived a fractional abundance of $10^{-5.8}$. This is based on the "standard" calibration method, with an estimated molecular hydrogen column density of $10^{24}$ $\mathrm{cm}^{-2}$, and is independent of the result derived from methanol maser excitation. For methyl formate we observed 12 lines and derived a fractional abundance of $10^{-7.1}$. For dimethyl ether, which we observed in 4 lines, we derived a fractional abundance of $10^{-7.0}$. All three fractional abundances are substantially higher than those reported by Helmich \& van Dishoeck (1997) at about 15 arcsec angular resolution (cf. Table 1).

Acknowledgments. This work was supported in part by the National Science Foundation under BIMA grant AST 99-81363.

\section{References}

Blake, G. A., Sandell, G., van Dishoeck, E. F., Groesbeck, T. D., Mundy, L. G., \& Aspin, C. 1995, ApJ, 441, 689

Blake, G. A., Sutton, E. C., Masson, C. R., \& Phillips, T. G. 1987, ApJ, 315, 621

Caselli, P., Hasegawa, T. I., \& Herbst, E. 1993, ApJ, 408, 548

Gibb, E., Nummelin, A., Irvine, W. M., Whittet, D. C. B., \& Bergman, P. 2000, ApJ, 545, 309

Goldsmith, P. F. \& Langer, W. D. 1999, ApJ, 517, 209

Helmich, F. P., Jansen, D. J., de Graauw, Th., Groesbeck, T. D., \& van Dishoeck, E. F. 1994, A\&A, 283, 626

Helmich, F. P. \& van Dishoeck, E. F. 1997, A\&AS, 124, 205

Ikeda, M., Ohishi, M., Nummelin, A., Dickens, J. E., Bergman, P., Hjalmarson, A., \& Irvine, W. M. 2001, ApJ, 560, 792

Macdonald, G. H., Gibb, A. G., Habing, R. J., \& Millar, T. J. 1996, A\&AS, 119,333

Nummelin, A., Dickens, J. E., Bergman, P., Hjalmarson, A., Irvine, W. M., Ikeda, M., \& Ohishi, M. 1998, A\&A, 337, 275

Nummelin, A., Bergman, P., Hjalmarson, Å., Friberg, P. Irvine, W. M., Millar, T. J., Ohishi, M., \& Saito, S. 2000, ApJS, 128, 213

Sutton, E. C., Jaminet, P. A., Danchi, W. C., \& Blake, G. A. 1991, ApJS, 77, 255

Sutton, E. C., Peng, R., Danchi, W. C., Jaminet, P. A., Sandell, G., \& Russell, A. P. G. 1995, ApJS, 97, 455

Sutton, E. C., Sobolev, A. M., Ellingsen, S. P., Cragg, D. M., Mehringer, D. M., Ostrovskii, A. B., \& Godfrey, P. D. 2001, ApJ, 554, 173

Sutton, E. C., Sobolev, A. M., Salii, S. V., Malyshev, A. V., Ostrovskii, A. B., \& Zinchenko, I. I. 2003, in preparation

van Dishoeck, E. F., Blake, G. A., Jansen, D. J., \& Groesbeck, T. D. 1995, ApJ, 447,760 